of the zero degree solution, namely

$$
y_{0}=\alpha_{r} \cos r \omega t+A_{1} \cos (n \omega t+\varphi), \quad A_{1}=A / \omega^{2}\left(r^{2}-n^{2}\right) .
$$

It should be emphasized that the preceding work has been carried out on the assumption that the forcing term $F(t)$ in (1) is of order unity. Synchronization effects have been sought for, therefore, only in the terms of the solution of this order of magnitude.

\title{
ReFERENCES
}

1. B. van der Pol, Phil. Mag. (7) 3, 65 (1927).

2. N. Minorsky, Introduction to non-linear mechanics, J. W. Edwards, Ann Arbor, Mich., 1947, especially p. 341.

3. H. G. Cohen, Actes du colloque international des vibrations non-linéaires, Ile de Proquerolles, 1951, P.S.T. 281, French Ministry of Air, 1953, pp. 169-188.

4. J. L. Stoker, Nonlinear vibrations, Interscience, New York, 1950.

\section{A NOTE ON THE PATTERSON FUNCTIONS*}

By C. R. PUTNAM (Purdue University)

The following brief note was suggested by a paper of Hartman and Wintner [1], dealing with the Patterson function $P(x)$, defined by

$$
P(x)=L^{-1} \int_{0}^{L} \rho(t) \rho(t+x) d t,
$$

where $\rho(x)$ denotes a positive periodic function of period $L$. If $\rho(x)$ possesses a finite number $N$ of (relative) maxima on the interval $0 \leqq x<L$, then, as a consequence of what the above authors refer to as the Patterson principle in crystallography (see [2]), the function $P(x)$ would have at most $N(N-1)$ maxima on the interval $0<x<L$. (Obviously, the function $P(x)$ is a positive even periodic function of period $L$, with absolute maxima occurring at the points $x=0, \pm L, \pm 2 L, \cdots$.) By constructing counter-examples for the case $N=2$, the authors show that $P(x)$ can have more than the predicted two peaks on $0<x<L$ and conclude that the Patterson principle cannot be valid as a general mathematical theorem.

The present note will deal with the case $N=1$, so that $\rho(x)$ corresponds, in the terminology of Patterson ([2], p. 521), to an electron density which is itself an "atomic function" possessing a single peak on $0 \leqq x<L$. It will be shown that not only is the Patterson principle (which would here deny the existence of any peaks of $P(x)$ on $0<x<L$; see loc. cit., middle of page 522) false even in this case, but that $P(x)$ may, in fact, have any specified finite number of peaks on $0<x<L$.

To this end, let $n$ denote an arbitrary positive integer and divide the interval $0 \leqq x \leqq L$ into $2 n$ parts of length $d=L / 2 n$. Next define the step-function $\rho(x)$ 
$(=\rho(x+L))$ on $0 \leqq x<L$ by:

$$
\rho(x)= \begin{cases}1, & 0 \leqq x<d, \\ k, & (2 k-3) d \leqq x<(2 k-1) d \quad(k=2, \cdots, n), \\ b, & (2 n-1) d \leqq x<2 n d,\end{cases}
$$

where $b$ is a positive constant to be determined. Then the graph of $P(x)$ is a polygonal path with vertices having abscissas on $0 \leqq x<L$ at $x=0, d, \cdots,(2 n-1) d$. It will be shown that for a proper choice of the constant $b$ the function $P(x)$ of (1) has maxima occurring at the points $x=0,2 d, \cdots,(2 n-2) d$. For reasons of continuity, it is clear that the above $\rho(x)$ can be replaced by a continuous positive function possessing a single maximum on the interval $0 \leqq x \leqq L$ and so that the corresponding $P(x)$ of (1) possesses $n$ maxima on $0 \leqq x<L$; see the remark of [1], p. 272 .

An easy calculation shows that, for the $\rho(x)$ of $(2)$, the function $P(x)$ satisfies

$$
P(m d)=c_{m}+\left\{\begin{array}{lll}
(n+1) b d, & \text { for } & m=1,3, \cdots, 2 n-1, \\
(n+2) b d, & \text { for } & m=2,4, \cdots, 2 n-2 .
\end{array}\right.
$$

Accordingly, since the constants $c_{m}$ do not depend on $b$, it is clear from (3) that for a sufficiently large $b$, the inequalities $P(2 k d)>P[(2 k+1) d]$ and $P[(2 k+1) d]<P[(2 k+$ $2) d]$ hold for $k=0,1,2, \cdots, n-1$. Thus, the function $P(x)$ has $n$ maxima on $0 \leqq x<L$ as claimed, and the existence of a continuous $\rho(x)$ with the desired properties now follows as remarked above.

\section{REFERENCES}

[1] P. Hartman and A. Wintner, On the maxima of the Patterson functions, Phys. Rev. 81, 271-273 (1951). [2] A. L. Patterson, A direct method for the determination of the components of interatomic distances in crystals, Z. Krist. 90, 517-542 (1935).

\section{ON HELICAL SPRINGS OF FINITE THICKNESS*}

\section{BY PETER HENRICI (National Bureau of Standards)}

1. Introduction. The problem of determining the stress distribution in a statically loaded, closely coiled helical spring, whose cross-section is not necessarily small in comparison with the diameter of the helix, has been solved approximately by iteration by Göhner $[5,6]$ and exactly in terms of a series of appropriate Legendre functions by Freiberger [4]. In the present paper it is shown that the exact values of the stress concentration factor as well as of some other significant quantities of the problem can be developed in terms of a certain parameter (namely the reciprocal of the so-called spring index) so as to recover Göhner's approximations and to obtain further approximations of the same type.

*Received May 20, 1954. The preparation of this paper was sponsored by the Office of Naval Research. 